027.7 Zeitschrift für Bibliothekskultur / Journal for Library Culture

\title{
Das virtuelle Büro - ein \\ neues Arbeitsmodell für \\ die Zeit nach der \\ Pandemie?
}

\section{Beat Mattmann}

Published on: Jul 27, 2021

License: Creative Commons Attribution 4.0 International License (CC-BY 4.0). 


\section{ABSTRACT}

Die Corona-Pandemie hat die Arbeitsweise in Bibliotheken innerhalb kürzester Zeit verändert - erzwungenermassen. Wie nachhaltig diese Veränderung hin zu mehr Virtualität ist, bleibt jedoch den Institutionen überlassen. Dass ein grösserer Anteil an Homeoffice nicht nur mit negativen Effekten verbunden ist, sondern Zufriedenheit und Produktivität von Mitarbeitenden sogar spürbar steigern können, zeigen verschiedene Studien. Der Erfolg der virtuellen Arbeit hängt aber letztlich zu einem guten Teil von der passenden Infrastruktur ab - von Kommunikation über die gemeinsame Arbeit an Inhalten bis hin zur strategischen Planung.

The Corona pandemic has changed the way libraries work in a very short time - of necessity. How sustainable this change towards more virtuality is, however, remains up to the institutions. Various studies show that a larger amount of home office work is not only associated with negative effects, but can even increase employee satisfaction and productivity. But the success of virtual work largely depends on the appropriate infrastructure - from communication to collaborative work on content up to strategic planning.

\section{Einleitung}

In den vergangenen Monaten wurden die meisten Menschen durch die CoronaPandemie und den damit einhergehenden Schutzmassnahmen der Behörden dem plötzlichen Umstand unterworfen, neue Wege der Zusammenarbeit finden zu müssen. Die Stimmen, die dies als Zwang erlebten und möglichst bald in die „Realität vor Corona“ zurückkehren wollten, konnten regelmässig in verschiedenen Medien und gar auf Demonstrationen vernommen werden - sowohl als Äusserungen von Einzelpersonen als auch von Unternehmen und Verbänden. Andere wiederum erlebten diese Zeit als eine Chance zur Kreativität, zum Sprung ins kalte Wasser der Welt von Tools und New Work Konzepten. Bereiche von Bibliotheken, die aufgrund ihrer Materie und Services bereits seit längerem einer hohen Dynamik unterworfen sind - zum Beispiel digitale Dienste und IT-Abteilungen grösserer Bibliotheken -, hatten gefühlt bereits einen gewissen Vorsprung an Erfahrung. Agile Frameworks und Konzepte, Nutzerorientierung, Flexibilität, ständige Lernbereitschaft - dies alles sind Ideen, die diese Bereiche bereits seit längerem beschäftigen (vgl. etwa den Artikel von Mattmann (2020) im Blog der Universitätsbibliothek Basel oder die Artikel von Lüthi (2020). und Weng (2021). auf dem Blog der Zentralbibliothek Zürich). Das rege Interesse der 
Bibliotheksbranche an diesen Themen illustrierte etwa auch ein Aufruf des Autors auf Twitter zur Mitwirkung an einer Community of Practice zu Agilität in Bibliotheken im Herbst 2020 1 oder die rege Teilnahme an Veranstaltungen der Interessengemeinschaft Wissenschaftliche Bibliothekar*innen Schweiz (IG WBS) im Januar 2021 (vgl. IG WBS, 2021). Gleichwohl gab und gibt es nicht nur positive Stimmen und die Zurückhaltung bei grösseren Veränderungen der Arbeitsweise muss ernst genommen werden. Dieser Artikel soll eine Übersicht über die verschiedenen Aspekte virtueller und verteilter Arbeit bieten und eine Auswahl an Hilfsmitteln und Tools vorstellen, zugleich aber auch skeptische Haltungen aufgreifen (vgl. Abbildung 1).

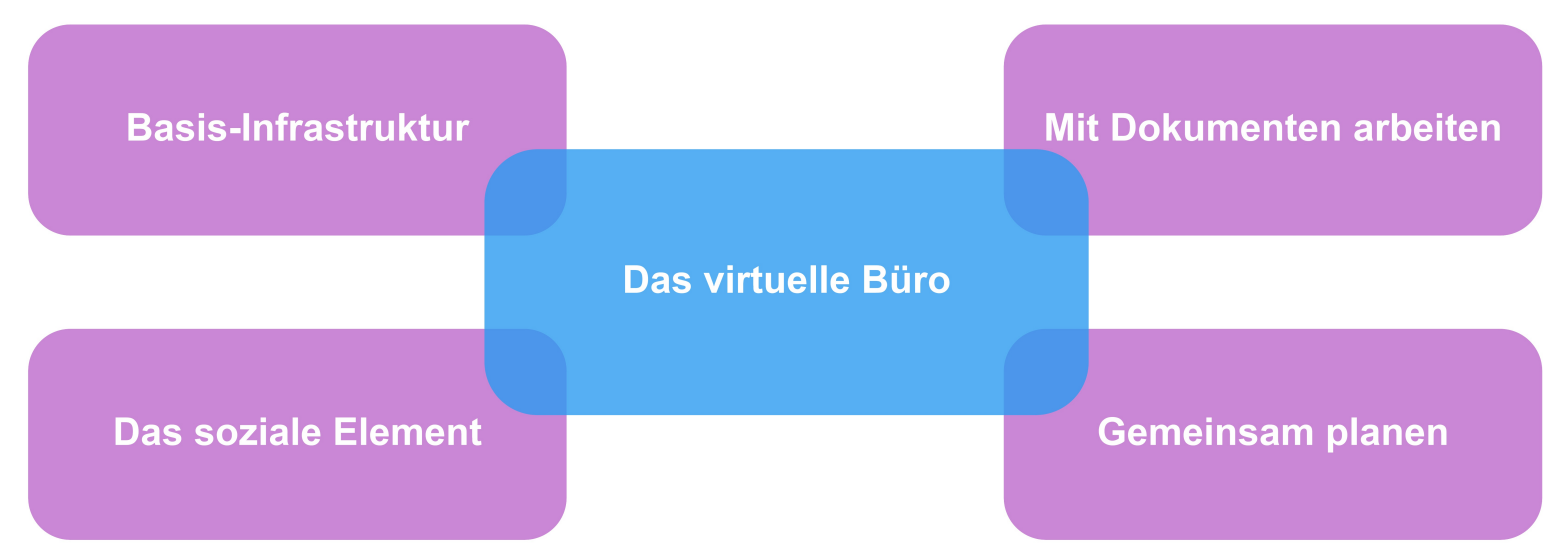

Abb. 1: Aspekte des virtuellen Arbeitens

\section{Kein Arbeiten ohne gute Grundlage}

Wer sich nicht mehr physisch treffen kann, aber dennoch Abstimmungsbedarf im Rahmen von Meetings hat, dem*der werden Tools wie Zoom, Microsoft Teams und Co. längst kein Fremdwort mehr sein. Gleichwohl: Wer in den vergangenen Monaten länger in virtuellen Meetings sass, wird irgendwann festgestellt haben, dass es gewisse Trigger gibt, die beinahe jeden stören, ungeachtet des Themas des Meetings: das Stocken von Ton und/oder Video, das dauerhaft ausgeschaltete Kamerabild einzelner Personen in einer grossen Runde sichtbarer Silhouetten, laute Hintergrundgeräusche, hämmernde Tastaturen usw. Dies lässt sich durchaus mit analogen Meetings vergleichen, in denen Teilnehmer*innen durch laute Störgeräusche (absichtlich oder unabsichtlich) auf sich aufmerksam machen, sehr leise oder sehr laut reden oder nebenbei deutlich vernehmbar auf ihrem Laptop tippen. In virtuellen Meetings müssen diese Störeffekte nicht einmal absichtlich auftreten. Aus Unerfahrenheit oder mangels Feedback sind sich viele des störenden Moments 
schlicht nicht bewusst. Hier empfiehlt sich ein offener Umgang und eine gemeinsam vereinbarte Etikette. Regeln wie die bewusste Aufmerksamkeit oder die Teilnahme mit Video (sofern das die Anzahl der Teilnehmer*innen und die Internetbandbreite gestatten) erlauben eine gleichberechtigte Teilnahme und die Vermittlung möglichst vielfältiger kommunikativer Information (durch Sprache, Gestik und Mimik). Zur Gewährleistung eines angenehmen und klaren Tons empfiehlt sich die Nutzung eines Headset statt des im Laptop oder Computer eingebauten Mikrofons. Zur Sicherstellung eines guten Bildes genügt es oft, direkt einfallende Lichtquellen im Hintergrund zu vermeiden oder sie so auszurichten, dass Lampen nicht direkt auf den Bildschirm beziehungsweise die Kamera gerichtet sind. Indirekte Lichtquellen und Tageslicht, das einen von vorne bescheint, unterstützen ein klares Bild ungemein. Sollte die Qualität immer noch zu schlecht oder keine eingebaute Kamera vorhanden sein, helfen externe Webcams weiter. Ein stockendes Bild oder zerhackter Ton wiederum lässt sich oft auf die Verbindungsgeschwindigkeit des WLANs vor Ort zurückführen. Hier lohnt sich unter Umständen die Wahl einer höheren Bandbreite oder der Wechsel des Anbieters.

Eine besondere Hürde stellt die hybride Kombination von analoger und virtueller Kommunikation dar. Wie kann man verschiedene Menschen in einem Raum vor Ort mit weiteren Personen, verteilt über verschiedene Standorte, virtuell verbinden? Auch dafür gibt es immer mehr Equipment. Ein charmantes Produkt ist etwa die Meeting Owl von Owl Labs - ein $360^{\circ}$-Mikrofon mit Richtkamera, die sich auf die aktuell sprechende Person fokussiert. $\underline{2}$

Das alles ist nicht etwa technisches Wunderwerk oder eine zu hohe, nicht zu verlangende Anforderung. Sie lässt sich vergleichen mit der Infrastruktur im Büro vor Ort. Wackelnde Stühle, fehlendes Equipment, ein langsames Netzwerk et cetera - auch im Büro gibt es störende Faktoren, die die Arbeit deutlich behindern. Sind diese Probleme jedoch erst einmal behoben, wird die gemeinsame Arbeit entspannter, das Denken gelöster und kreativer und die Mitarbeiter*innen insgesamt zufriedener. Denn oft sind es bereits die kleinen Dinge, die eine grosse Wirkung erzielen können und dennoch vergessen werden. Natürlich, Equipment ist nicht immer billig und nicht jede*r verfügt über das Budget, technische Geräte mit stimmiger Qualität anzuschaffen. An dieser Stelle sollten die Arbeitgeber*innen kulant und um eine gute Ausrüstung ihrer Mitarbeitenden besorgt sein - zumindest hinsichtlich Kommunikationsmitteln und Arbeitsgeräten wie Laptops. 


\section{Die gemeinsame Arbeit an Dateien}

Die heutige Welt der Wissensarbeit funktioniert überwiegend über Texte und Tabellen

- Dokumente und Dateien. Manchmal noch auf Papier, immer mehr aber über digitale Dateiablagen. Vielfach haben sich hier Dateiablagesysteme wie GEVER etabliert, ermöglichen sie doch gleichzeitig die spätere Archivierung von Dokumenten gemäss der für Verwaltungen geltenden Archivgesetze. $\underline{3}$ Klassische Netzwerk-Dateiablagen ermöglichen aber noch keine synchrone Kollaboration. Hat jemand ein Dokument geöffnet, können andere in der Regel nur im Lesemodus darauf zugreifen. Des Weiteren sind die klassischen lokalen Ablagen nicht für Unternehmensexterne zugänglich, was bei institutionsübergreifenden Arbeitsgruppen und Kooperationen eine gewisse Hürde darstellt. Stattdessen werden Dokumente meist in unzähligen Versionen per Mail hin und her geschickt, wodurch beinahe zwangsläufig irgendwann die Übersicht verloren geht, welche Version gerade aktuell ist. Einfacher sind da digitale Arbeitsablagen, die die kollaborative und zeitgleiche Arbeit an Dokumenten erlauben - in der Regel mit integrierter Office-Suite und Versionshistorie. Das können bekannte kommerzielle Anbieter wie Google Drive $\underline{4}$ sein (die in vielen Verwaltungen aber aus Datenschutz- und rechtlichen Gründen entweder umstritten oder verboten sind), aber auch Anbieter aus dem Verwaltungs- und Hochschulbereich. So bietet in der Schweiz zum Beispiel SWITCH$\underline{5}$, eine Stiftung mit einem Angebot digitaler Infrastrukturen für die Bildungs- und Forschungscommunity, eine digitale Dateiablage namens SWITCHdrive $\underline{\underline{6}}$ mit integrierter Office-Suite (OnlyOffice) an. Der Zugang erfolgt über das Identity Management der Hochschulen, wobei jede*r Nutzer*in 100 GB an Speicherplatz zur Verfügung hat.

Wer es gerne etwas elaborierter hätte, kann auf Wissensmanagement-Lösungen wie Atlassian Confluence, DokuWiki, XWiki oder BlueSpice zurückgreifen. $\underline{7}$ Neben den klassischen Wiki-Funktionalitäten wie editierbaren Seiten mit Zugriffssteuerung und Versionierung lassen sich meist auch Plugins einbinden, die eine solche Lösung erst richtig mächtig machen können. So können etwa Grafiken, Schemas und Skizzen direkt eingebunden und editiert werden (beispielsweise mittels draw.io, Mural oder Miro) oder eine Verknüpfung zu Planungstools wie Trello oder Atlassian Jira herstellen. $\underline{8}$ Wie immer gilt auch hier die Abwägung im Sinne von Fragen des Datenschutzes - wobei es mittlerweile Lösungen für verschiedenste Bedürfnisse gibt. 


\section{Planung im Grossen und Kleinen}

Ein grosses Thema bei der Zusammenarbeit ist die Planung - Strategien, Projekte, die alltägliche Abstimmung. Da genügt oft schon der persönliche Austausch (worauf später noch eingegangen wird), mehr aber noch müssen Roadmaps und Vorgehensweisen festgelegt und transparent gemacht, Arbeitspakete definiert und ausgearbeitet oder Zuständigkeiten besprochen werden. In der analogen Welt noch oft an Whiteboards erfolgt, mit unzähligen Post-Its an Wänden oder auch klassisch in Word- und Excel-Dokumenten in der Dateiablage, bieten sich in der rein virtuellen Welt viele Tools als mächtige Unterstützung an, insbesondere aus der agilen Welt. $\underline{9}$ Das bedeutet jedoch nicht, dass man mit solchen Instrumenten zugleich eine agile Transformation anstösst! Sie lassen sich unabhängig davon für die Planung der eigenen Aufgaben oder jener ganzer Teams oder Abteilungen einsetzen. So ermöglicht etwa Trello mit Elementen aus Kanban die Aufteilung von Projekten in einzelne Aufgaben inklusive Aufgabenbeschreibung, Kommentaren und Anhängen, Fristen und der Zuweisung zu einzelnen Personen. 10 Über mehrere Spalten hinweg können diese Aufgaben verschoben werden und so transparent machen, in welchem Status sie sich gerade befinden (zum Beispiel geplant, in Arbeit, wartend auf bestimmte Aktionen, erledigt ...). Auch für die täglichen Aufgaben lässt sich diese Methode nutzen (oder im Privaten für Urlaubs- und Umzugsplanungen, das Homeschooling der Kinder und so weiter). $\frac{11}{11}$

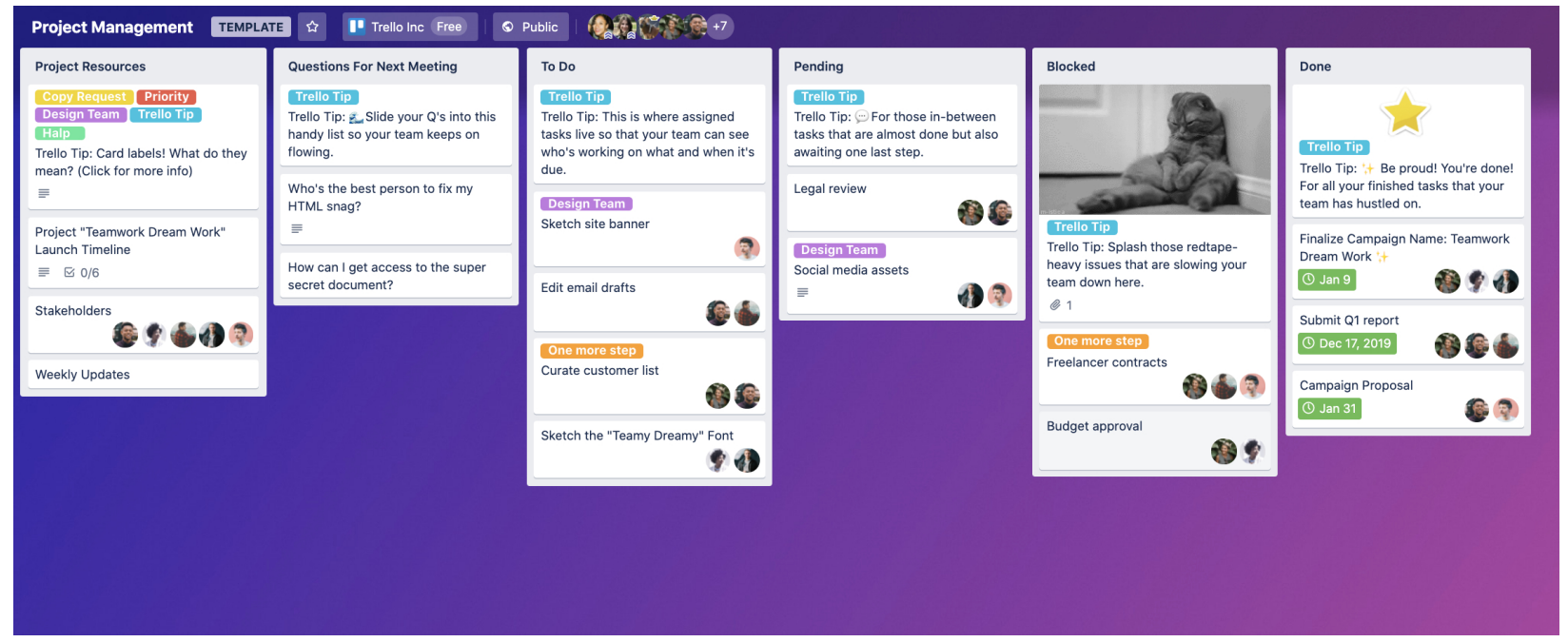

Abb. 2: Beispiel eines Trello-Boards für das Management eines Projekts (Grafik entnommen aus Mesh (2020)).

Natürlich ist Trello nicht alleiniger Anbieter von Tools solcher Art. Weitere sind etwa Atlassian Jira, Kanbanize oder Meistertask. $\underline{12}$ Darüber hinaus gibt es Tools, die 
Funktionalitäten der Projektplanung um Kommunikationselemente, Videokonferenzen und Dateiablagen ergänzen, wie etwa Stackfield $\underline{13}$, welches als deutsches Unternehmen insbesondere mit Datenschutz nach europäischen Vorgaben wirbt. Dadurch entsteht ein beinahe umfassendes virtuelles Büro, das alle benötigten Instrumente an einem Ort zusammenführt und miteinander verbindet. Derzeit nutzt etwa die Zentral- und Hochschulbibliothek Luzern Stackfield als zentrales Kollaborations- und Kommunikationstool.

\section{Das soziale Element}

Was viele im virtuellen Büro wohl am meisten vermissen dürften, ist das soziale Element - der kurze Austausch am Kaffeeautomaten, gemeinsame Pausen, der gelegentliche Smalltalk oder die kurze Frage über den Bürotisch. In dieser Einfachheit und Gewohnheit wird kein virtuelles Tool dies ersetzen können. Es gibt jedoch durchaus Möglichkeiten, Alternativen zu kreieren und Gelegenheit zu Austausch und Beziehungspflege zu bieten. Klassisch und mittlerweile bekannt dürften hier virtuelle Kommunikationstools wie Zoom oder Microsoft Teams sein, in Institutionen mit rigideren Datenschutzvorschriften alternativ auch Webex oder BigBlueButton. $\underline{14}$ Viele nutzen diese Tools aber nur in ihrer minimalsten Form: als Plattform für Videokonferenzen. Darüber hinaus bieten sie aber meist auch Möglichkeiten für Chats, sowohl bilateral als auch für Teams oder Büros. Darin lässt sich sowohl die „kurze Frage zum Nachbartisch“ stellen, als auch zwischendurch zur Unterhaltung ein Bild, GIF oder ein Gedanke teilen. Ein Vorteil gegenüber der analogen Welt ist jedoch, dass der oder die Angesprochene die Möglichkeit hat, zu steuern, wann man reagieren möchte - was insbesondere bei mitteilsamen Bürokolleg*innen ein nicht zu unterschätzender Gewinn ist. Es lassen sich zudem gezielt Chaträume für einzelne Projekte oder Arbeitsgruppen erstellen. Allerdings sind diese teilweise begrenzt auf Nutzer*innen aus der eigenen Institution. Für den institutionsübergreifenden Austausch und Abstimmungsbedarf gibt es Alternativen wie Slack oder Discord. $\underline{15}$ Gerade Letzteres zeigt seine Stärke darin, dass neben individuell gestaltbaren Chaträumen auch solche für Audio- und Videokonferenzen möglich sind. Es entfällt jedoch das Sharen von Links, wie dies etwa Zoom erfordert. Vielmehr können Räume so konfiguriert werden, dass jede*r Teilnehmer*in des Discord-Servers spontan dazu stossen kann - das wohl treffendste virtuelle Pendant zum Kaffeeautomaten. Dass das durchaus auch grössere Dimensionen annehmen kann und darf, zeigen etwa Beispiele von Discord-Servern für die Vernetzung von Bibliotheksmitarbeitenden. $\underline{16}$ Natürlich ist auch hier die Frage des Datenschutzes präsent. Wenn es um den einfachen Austausch 
geht, kann diese nach Ansicht des Autors jedoch durchaus entspannter gesehen werden - zumal etwa Discord zwecks Identifikation lediglich ein Pseudonym und eine E-Mail-Adresse verlangt. Sollen jedoch auch heiklere Themen besprochen werden können, sollte auf Alternativen aus dem Open Source Bereich, wie etwa Matrix, ausgewichen werden. $\underline{17}$

\section{Exkurs: Kritik an der virtuellen Arbeit}

Die Erfahrungen, die mit der erzwungenen virtuellen Arbeitsweise von Zuhause seit Beginn der Pandemie gemacht wurden, kann man durchaus als zwiespältig bezeichnen. Davon zeugen etwa die verschiedenen Meinungsäusserungen führender Personen grösserer Unternehmen in der Zeitschrift „vitra“ (Vitra, 2021), aber auch eine Studie des Beratungsunternehmens Von Rundstedt, die unter 534 Schweizer Unternehmen aus verschiedenen Branchen und Sprachregionen durchgeführt wurde. $\underline{18}$ Die Skepsis der Führungsebene gegenüber dem Arbeiten von Zuhause aus scheint zwar gross (vgl. Von Rundstedt,_2021,8-9 und 19-20), gleichzeitig widersprechen die Ergebnisse der Studie dieser Skepsis teilweise. So scheint das Vorurteil, dass die Produktivität der Mitarbeitenden im Homeoffice sinkt, nicht zuzutreffen. Eine Mehrheit der befragten Führungspersonen beurteilt die Produktivität und die Arbeitsqualität nach einem Jahr Corona-Homeoffice als höher oder gleichbleibend (Von Rundstedt 2021,12-13). Interessant sind die Erkenntnisse zum optimalen Anteil des Remote Workings an der Gesamtarbeitszeit: Verschiedene Untersuchungen von Golden und Veiga (2005), Golden (2006). und Derks und Bakker (2014). erachten aus Sicht von Work-Life-Balance, Beziehungsqualität und Motivation einen Anteil von zwei Arbeitstagen ausserhalb des Unternehmens als optimal, alles darüber hinaus kann in den genannten Aspekten einen negativen Effekt auf Mitarbeitende haben. Das Ideal ist also, wie so oft, die Balance: Die Flexibilisierung der Arbeit hat sowohl positive Effekte auf Produktivität, Qualität und Zufriedenheit der Mitarbeitenden, ein gewisser Anteil an Vor-Ort-Arbeit erhält beziehungsweise stärkt wiederum das Wir-Gefühl.

Natürlich hängt die Zufriedenheit von Mitarbeitenden auch von weiteren Faktoren ab. So beleuchtet eine Studie der Fachhochschule Nordwestschweiz und der Zürcher Hochschule für Angewandte Psychologie von Schulte et al. Aspekte des Wohlbefindens von Mitarbeitenden im Homeoffice. Die Studie führte eine nicht repräsentative Befragung unter 333 Personen aus verschiedenen Dienstleistungsbereichen durch und stellte grundsätzlich ein hohes Wohlbefinden im Homeoffice fest. Ausschlaggebende förderliche Faktoren sind laut Schulte et al. (2020,23-24) etwa eine gute Führung durch vorgesetzte Personen, klare Kommunikationsregeln sowie eine grosse 
persönliche Autonomie bei gleichzeitiger enger Einbindung in das Team. Als negativer Einfluss empfunden wurde hingegen der mangelnde soziale Austausch.

\section{Virtuelles Arbeiten als neue Arbeitsweise nach Corona?}

Was wäre nun die „richtige“ Arbeitsweise für die Zeit nach Corona? Gibt es sie überhaupt? Wie so oft ist die Bewertung der „Richtigkeit“ eine Sache von Auslegung und Präferenzen. Die Frage, die sich Bibliotheken stellen sollten, ist, ob eine flexiblere Arbeitsweise und ein grösserer Anteil an virtueller Arbeit die Zufriedenheit der Mitarbeitenden generell steigern und gleichzeitig die Produktivität und Arbeitsqualität erhalten kann. Wirft man einen Blick in die hier vorgestellten Studienergebnisse (vgl. den vorherigen Abschnitt), so kann diese Frage durchaus positiv beantwortet werden, sofern die Balance stimmt. Gestaltet man die virtuelle Arbeit zudem so, dass effizient und hindernisarm gearbeitet werden kann - durch gutes Equipment sowie geeignete Hilfs- und Kommunikationsmittel -, entsteht ein hybrides Büro, das vielen Bedürfnissen zugleich begegnen kann. Unser Arbeitsalltag dürfte sich durch die lange Zeit der Pandemie vielerorts sichtbar verändert haben, auch wenn das zu diesem Zeitpunkt noch Spekulation bleiben muss. Solche Spuren sind aber bei Weitem nichts Negatives! Vielmehr bieten sie Lösungen und Chancen, um zugleich bereits vor Corona vorhandenen Entwicklungen und Schwierigkeiten in der Arbeitswelt zu begegnen. Findet beispielsweise die Kommunikation hybrid statt - also sowohl analog vor Ort als auch virtuell in Teamräumen -, so bietet sich Eltern die Möglichkeit, mit vermehrtem Homeoffice die Kinderbetreuung zu erleichtern. Dasselbe gilt für die Pflege von Angehörigen. Verschiebt man die gemeinsame Arbeit an Dokumenten ganz in die virtuelle Welt und in kollaborative Tools, reduzieren sich Missverständnisse und Versionschaos bei digitalen Unterlagen auf ein Minimum. Finden Konferenzen und Treffen für grössere Publikumskreise hybrid oder virtuell statt, reduzieren sich die CO2-Emissionen für weite Reisen und zugleich haben erstmals auch Unternehmen mit kleinem Budget oder Menschen mit körperlichen Beeinträchtigungen oder begrenzten zeitlichen beziehungsweise finanziellen Ressourcen die Möglichkeit, an der (inter-)nationalen Vernetzung teilzunehmen und neue Inspirationen zu erhalten. Insgesamt vergrössert sich somit der Reichtum an Möglichkeiten und die Flexibilität der Arbeitsweise. Das virtuelle Büro ist also nicht nur eine, durch eine Pandemie erzwungene, temporäre Veränderung, sondern vor allem auch eine grosse Chance sofern man sie denn wahrnehmen möchte.

\section{Footnotes}

1. Vgl. https://twitter.com/BeMattmann/status/1292016640218075136 
2. Vgl. https://owllabs.com/. $\doteq$

3. GEVER, Abkürzung für GEschäftsVERwaltung, dient in der Schweiz als Akronym für die elektronische Aktenführung im Verwaltungsbereich. Siehe dazu auch https://de.wikipedia.org/wiki/GEVER. $\doteq$

4. Vgl. https://www.google.ch/drive/. $ヒ$

5. Vgl. https://www.switch.ch/.

6. Vgl. https://www.switch.ch/drive/.

7. Eine gute Übersicht über aktuelle Angebote inklusive Bewertung findet sich in der Februar-Ausgabe von iX, vgl. Loschwitz, M. G. (2021). Wissen ist Macht. Marktübersicht: Was Knowledge-Management-Systeme leisten. iX, (2). Retrieved from https://www.heise.de/select/ix/2021/2/2028911453562760368. Sie enthält sowohl proprietäre als auch Open Source Angebote, cloudbasierte und lokal installierbare Lösungen. Beschreibungen zu der genannten Auswahl, vgl. https://www.atlassian.com/software/confluence, https://www.dokuwiki.orgL, https://www.xwiki.org $L$ https://bluespice.com/. $\doteq$ 8. Vgl. https://www.draw.io/, https://www.mural.co/, https://miro.com/, https://trello.com/, https://www.atlassian.com/de/software/jira.. 9. Eine kurze Einführung in das Thema der Agilität für Bibliotheken bietet Mattmann, B. (2020). Was ist eigentlich diese Agilität, von der alle reden? Retrieved from https://www.igwbs.ch/was-ist-eigentlich-diese-agilitaet-von-der-alle-reden/ bzw. https://doi.org/10.5451/unibas-ep80673.

10. Eine übersichtliche Einführung in die Idee von Kanban bietet https://kanbanize.com/de/kanban-ressourcen/kanban-erste-schritte..

11. Einen Überblick über die Methode und mögliche Anwendungsfälle hat Trello selbst in ihrem Blog beschrieben, vgl. Mesh, J. (2020). Mehr Agilität für jedes Team durch die Kanban-Methode. Retrieved from https://blog.trello.com/de/kanban$\underline{\text { methode. }}$.

12. Vgl. https://kanbanize.com/, https://www.meistertask.com/.. 13. Vgl. http://stackfield.com/. 
14. Vgl. https://zoom.us/, https://www.microsoft.com/microsoft-teams/, https://www.webex.com/, https://bighluebutton.orgL.

15. Vgl. https://slack.com/, https://discord.com/. Aber auch bereits erwähnte Tools wie Stackfield, Zoom oder Teams bieten die Möglichkeit, externe Personen in Chatoder Kollaborationsräume einzuladen - mit jeweils mehr oder weniger hohen Hürden.

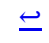

16. So erstellte eine Gruppe engagierter Bibliotheksmitarbeiter:innen, darunter auch der Autor, einen Discord-Server „DACH Bibliothekswesen”, der innert weniger Wochen mehrere hundert Bibliotheksmitarbeitende versammeln konnte. Ein weiteres Beispiel ist der Discord-Server „Gamebrarians” für Bibliotheksmitarbeitende mit einer Leidenschaft für Videospiele. $\leftrightarrows$

17. Vgl. https://matrix.org $L \cdot$.

18. Die Studie ist nicht absolut repräsentativ, da der Anteil an Antworten von grösseren Unternehmen mit 57\% (Unternehmen mit mehr als 250 Mitarbeitenden) höher ausfällt als jener von KMUs und die Antworten daher die Arbeitsrealität nicht exakt abbilden. $ヒ$

\section{Citations}

1. Mattmann, B. (2020). UB-Blog: Agile Methoden in Bibliotheken. UB Basel Blog. Retrieved from https://blog.ub.unibas.ch/2020/12/08/ub-blog-aggile-methoden-inbibliotheken/ $\triangleq$

2. Lüthi, C. (2020). Produktentwicklung mit Scrum. ZBlog. Retrieved from https://www.zb.uzh.ch/de/news/zblog\#scrum $\leftrightharpoons$

3. Weng, A. (2021). Wie die ZB Innovation fördert. ZBlog. Retrieved from https://www.zb.uzh.ch/de/news/zblog\#innovation $\leftrightharpoons$

4. IG WBS. (2021). Spread the word - Agilität und Bibliotheken, das funktioniert! (IGWBS Veranstaltung vom 6. und 27. Januar 2021). Retrieved from https://www.igwbs.ch/spread-the-word-agilitaet-und-bibliotheken-das-funktioniert-ig= wbs-veranstaltung-vom-6-und-27-januar-2021/

5. Vitra. (2021). Distributed Work. Vitra. The e-Paper about the Future of Shared Spaces, (5). Retrieved from https://www.vitra.com/de-ch/sessions/distributed-work $\triangleq$ 
6. Von Rundstedt. (2021). Smart Working in der Schweiz. Wie viel hat sich seit Beginn des Lockdowns wirklich verändert? Retrieved from https://www.rundstedt.ch/wp-content/uploads/2021/03/Whitepaper_SmartWorking_2021 DE-1.pdf $\leftrightarrows$

7. Golden, T. D., \& Veiga, J. F. (2005). The Impact of Extent of Telecommuting on Job Satisfaction: Resolving Inconsistent Findings. Journal of Management, 31(2), 301318. https://doi.org/10.1177/0149206304271768

8. Golden, T. D. (2006). The role of relationships in understanding telecommuter satisfaction. Journal of Organizational Behaviour, 27(3), 319-340. https://doi.org/10.1002/job.369

9. Derks, D., \& Bakker, A. B. (2014). Smartphone Use, Work-Home Interference, and Burnout: A Diary Study on the Role of Recovery. Applied Psychology, 63(3), 411-440. https://doi.org/10.1111/j.1464-0597.2012.00530.x

10. Schulte, V. B., Steinebach, C., Verkuil, A. H., \& Hübenthal, S. (2020). Studie zur Umstellung auf Homeoffice in der Schweiz während der Covid19 Krise. Retrieved from https://www.fhnw.ch/de/die-fhnw/hochschulen/hsw/media-newsroom/news/diemehrheit-fuehlt-sich-wohl-im-homeoffice/media/studie-erhebung-umstellung-aufhomeoffice-17042020. $\underline{\text { df }} \leftrightharpoons$ 